\title{
Impaired parent-reported health-related quality of life of underweight and obese children at elementary school entry
}

\author{
Amy van Grieken • Lydian Veldhuis • \\ Carry M. Renders · Jeanne M. Landgraf • \\ Remy A. Hirasing • Hein Raat
}

Accepted: 17 May 2012/ Published online: 14 June 2012

(C) The Author(s) 2012. This article is published with open access at Springerlink.com

\begin{abstract}
Purpose Examine the health-related quality of life of 5-6-year-old underweight, overweight and obese children. Methods Our cross-sectional study included 3,227 parentchild dyads from the "Be active, eat right" study. Parents completed questionnaires regarding child and parental characteristics. Health-related quality of life of the child was measured using the Child Health Questionnaire Parent Form 28. Children were classified normal weight, overweight, obese, severely obese, and underweight according to the international age and gender BMI cutoff points. Bootstrap analyses were performed for general linear models corrected for potential confounding variables.

Results Severely obese children $(\beta,-2.60 ; 95 \%$ CI, -4.80 to $-0.57, p<0.01)$ and underweight children $(\beta,-1.11 ; 95 \%$ $\mathrm{CI},-1.85$ to $-0.39, p<0.01)$ had lower parent-reported scores
\end{abstract}

Electronic supplementary material The online version of this article (doi:10.1007/s11136-012-0211-x) contains supplementary material, which is available to authorized users.

A. van Grieken · L. Veldhuis · H. Raat $(\bowtie)$

Department of Public Health, Erasmus MC University Medical Center, P.O. Box 2040, 3000 CA Rotterdam, The Netherlands

e-mail: h.raat@erasmusmc.nl

\section{M. Renders}

Institute of Health Sciences, Faculty of Earth and Life Sciences and EMGO Institute for Health and Care Research, VU

University Amsterdam, Amsterdam, The Netherlands

J. M. Landgraf

HealthActCHQ Inc., Cambridge, MA, USA

R. A. Hirasing

Department of Public and Occupational Health, EMGO Institute for Health and Care Research, VU University Medical Center, Amsterdam, The Netherlands on the physical summary scale. On the physical functioning profile scale parents of overweight and severely obese children also reported statistically significant lower scores $(p<0.05$ and $p<0.01$, respectively).There were no significant differences regarding the psychosocial summary scale scores between the different weight categories.

Conclusion Underweight and overweight children experience impaired health-related quality of life on the physical functioning domain. Physicians, teachers and parents should be aware of the possible negative impact on healthrelated quality of life in underweight and overweight 5-6year-old children.

Keywords Underweight - Overweight and obesity · Children · Health-related quality of life - CHQ-PF28

\author{
Abbreviations \\ BMI Body Mass Index \\ CHQ-PF28 Child Health Questionnaire Parent Form 28
}

\section{Introduction}

According to a recent study, the global prevalence of overweight (including obesity) among preschool children (0-5 years) has increased from $4.2 \%$ in 1990 to $6.7 \%$ in 2010: If this trend continues, a prevalence of $9.1 \%$ is projected in 2020 [1]. In the Netherlands, the prevalence of overweight and obesity among children and adolescents (age, 2-21 years) in 2009 was estimated at $12.8 \%$ for boys and $14.8 \%$ for girls [2].

Being overweight and/or clinically obese in childhood can have profound physical and psychosocial consequences (e.g., diabetes, hypertension and negative self-evaluation), which impact the everyday health-related quality of life for 
children and adolescents [3, 4]. Overweight adults have reported more limitations on the physical dimension of health-related quality of life compared to normal weight adults [5, 6]. Also, among overweight and obese adolescents and older school-aged children (10-18 years), decrements have been reported for both the physical and psychosocial dimensions of health-related quality of life [7-19]. However, the few studies that have reported on the association between health-related quality of life and being overweight among children (4-7 years) [20, 21] have reported contradicting results. Wake et al. [20] reported that health outcomes were similar for normal weight and obese children, whereas Skinner et al. [21] reported that parents of very obese children reported decreases in general health and limitations in activity.

The prevalence of underweight in children (2-6 years) was estimated as $13 \%$ for boys and $12 \%$ for girls in the Netherlands in 1997 [22]. An impact on health-related quality of life has been reported among underweight adults [23]. However, studies that have examined the association between health-related quality of life and being underweight in children and adolescents reported more mixed results [11, 20, 24-27].

At the age of 5-6 years when children enter elementary school, any limitations with regard to physical and psychosocial well-being and therefore health-related quality of life could impede a child's start to his or her school career [28-30]. The aim of this study was to compare the healthrelated quality of life for 5-6-year-old children who are underweight, overweight, and obese relative to age-matched peers of normal weight by means of the Child Health Questionnaire Parent Form 28 (CHQ-PF28) [31, 32]. Our hypothesis was that the health-related quality of life of underweight, overweight and obese children would be lower (i.e., worse) relative to age-matched children of normal weight.

\section{Methods}

\section{Study population}

The present cross-sectional study used data obtained at enrollment in the "Be active, eat right" study, a cluster randomized controlled trial that aims to assess the effects of an overweight prevention protocol as described in detail elsewhere [33]. The Medical Ethics Committee of the Erasmus MC (University Medical Centre Rotterdam) approved the study protocol (reference number MEC-2007163). A total of 13638 parents visiting one of the 44 participating Youth Health Care centers for their 5-year-old child's regular preventive health check between 2007 and 2008 were invited to participate in the study. The Youth
Health Care centers sent parents the regular invitation to come to the center for the child's regular preventive health check; information on the study, an informed consent form and the first questionnaire on child and parental characteristics was included with the invitation. Of the parents who presented for their child's health check, $64.4 \%$ provided informed consent $(n=8,784)$ for participation in this study and $98.9 \%(n=8,683)$ of the parents with consent returned the first questionnaire. A second questionnaire, including the CHQ-PF28, was provided to all study participants visiting 22 Youth Health Care centers $(n=3,942)$ (these families were not eligible to receive an intervention) and to study participants with overweight or obesity in the remainder 22 Youth Health Care centers $(n=439)$ (these families were eligible to receive an intervention). The Youth Health Care centers were randomly assigned to either provide an intervention or not, in the context of the "Be active, eat right" study. Not all parents received the second questionnaire in order to minimize respondent burden. The response rate to the second questionnaire was $74.8 \%$ (Figure 1 in the supplementary material shows a flow chart of the data collection process).

A study population of 3,227 children remained after removing records with missing data on child's gender, weight, height or all CHQ-PF28 items $(n=51)$.

\section{Weight status of the child}

Each child's weight and height was measured by trained Youth Health Care professionals using standardized methods during the regular preventive health check [34]. Body mass index (BMI) was calculated by the researchers as weight in kilograms divided by height in meters squared. Children were categorized into one out of five weight categories according to their BMI as follows: underweight, normal weight, overweight (not (severe) obesity), obesity (not severe obesity) or severe obesity. The international age-specific and gender-specific BMI cutoff points were applied to categorize children into underweight, normal weight, overweight and obesity [35, 36]. Obese children were categorized into obese (not severely obese) and severely obese. There are currently no international BMI cutoff points for severe obesity for children, but based on recent literature [37] and sample size considerations, we used the following cutoff points to categorize severe obesity: for boys, BMI $\geq 20.00$; for girls, BMI $\geq 21.00$.

Health-related quality of life

Health-related quality of life was assessed by means of the CHQ-PF28. There are 28 items on the CHQ-PF28 with four, five or six response options across eight multi-item scales and five single-item concepts. For the interpretation 
of the CHQ-PF28 scale scores and the psychometric properties of the CHQ-PF28 scales in the present study please see Table 1. As per the standardized developer instructions, the items from each of the scales were summed (some recoded/recalibrated) and transformed into 0 (worst possible score) to 100 (best possible score) scale [38]. From these "profiles," it is also possible to compute a two-dimensional summary known as the physical and psychosocial summary scales [32]. Both summary scales were considered the main outcomes; the profile scales were analyzed in an exploratory way [39].

Child and maternal characteristics

Information on child gender (male and female), age (years), ethnicity (Dutch, non-Dutch) and chronic health conditions was obtained at enrollment (Table 2). Child ethnicity (Dutch, non-Dutch) was determined based on the parents' country of birth: If both parents were born in the Netherlands, the child was classified "Dutch", and otherwise, the child was classified "non-Dutch" [40]. The prevalence of common chronic conditions was assessed and included asthma, hearing difficulties, seeing difficulties, abdominal pain, headaches or migraine, allergies, and eczema.

The majority of the questionnaires were completed by mothers $(89.3 \%)$. Information on maternal age (years), height (meters), weight (kilograms), country of birth (Dutch, non-Dutch), educational level (low, mid-low, midhigh, and high) and marital status (married/ cohabiting or single) was obtained at enrollment. Maternal BMI was calculated as weight in kilograms divided by height in meters squared. Weight status of the mother was categorized into one of the two categories based on BMI: no overweight $(\mathrm{BMI}<25)$ or overweight $(\mathrm{BMI} \geq 25)$ [41]. Maternal level of education was categorized into one of the four levels: low (no education, primary school, or $\leq 3$ years of general secondary school), mid-low ( $>3$ years of general secondary school), mid-high (higher vocational training, undergraduate programs, or bachelor's degree), and high (higher academic education) [42].

\section{Analyses}

Normal weight, overweight, obese, severely obese and underweight children were compared by means of one-way analysis of variance on mean age, average number of chronic health conditions and maternal age. Chi-square tests were performed to compare child (gender, ethnicity, number of chronic health conditions) and maternal characteristics (country of birth, educational level, marital status, weight status) among the weight categories.

Taking into account the non-normal distribution of some of the CHQ-PF28 scale residuals, nonparametric tests were performed to compare scale outcomes across weight categories. The normal weight children were considered the reference group in all analyses. Kruskal-Wallis tests were used to compare the CHQ-PF28 scale scores across weight categories; a significant test $(p<0.05)$ provides statistical support for performing pairwise comparisons [39]. MannWhitney U-tests were used to compare the CHQ-PF28 scale scores pairwise; each subgroup was compared with the reference group of normal weight children.

General linear models were fitted to study the association between child weight status and health-related quality of life as measured by the CHQ-PF28 scales. The CHQPF28 scales were studied as dependent variables, and the weight categories were studied as determinants, with the normal weight children as reference group. Adjusted general linear models were fitted to correct for potentially confounding variables; both child (age, gender, ethnicity, and number of chronic health conditions) and maternal variables (age, BMI, country of birth, education and marital status) were included. Following the suggestions made by Griffiths et al. [18], an interaction term was introduced to the model to examine whether there were differences in the association between weight status and health-related quality of life for boys and girls and Dutch and non-Dutch children. Associations and interaction terms were evaluated at $p<0.05$ level. Significant interactions were found for both gender and ethnicity. Statistical analyses were performed with SPSS 18.0 (SPSS Inc., Chicago, IL).

Due to the non-normal distribution of some of the CHQPF28 scale residuals, the bootstrap procedure was applied to estimate the regression coefficients of the general linear models [43, 44]. Both the unadjusted (uncorrected for potentially confounding variables) and the adjusted (corrected for potentially confounding variables) bootstrap sampling results are reported with $95 \%$ confidence intervals (95\% CI) and coefficient $p$ values. The bootstrap procedure was conducted in $\mathrm{R}$ version 2.7.1 ( $\mathrm{R}$ Development Core Team 2008).

\section{Results}

The mean age of the children in the sample was 5.7 [SD: $0.4]$ years. Within the sample, $49.3 \%$ were boys. Of all children in the sample, $37.6 \%$ had one or more chronic health conditions. There were significantly more Dutch children in the underweight and normal weight categories compared to the percentage Dutch children in the overweight, obese and severely obese categories $(p<0.001)$. The overall mean BMI of the children in the study sample was 15.7 [SD: 1.7], and the mean BMI of the children with normal weight was 15.5 [SD: 0.9]. There were more boys than girls within the subgroups of severe obesity $(66.7 \%)$ 
Table 1 CHQ-PF28 scales, number of items per scale, score interpretation and psychometric properties in the present study $(n=3,227)$

\begin{tabular}{|c|c|c|c|c|c|c|c|c|c|c|}
\hline \multicolumn{4}{|c|}{ CHQ-PF28 scales } & \multicolumn{7}{|c|}{ Overall $(n=3,227)$} \\
\hline & $\begin{array}{l}\text { Number } \\
\text { of items }\end{array}$ & $\begin{array}{l}\text { Description } \\
\text { low score }\end{array}$ & $\begin{array}{l}\text { Description } \\
\text { high score }\end{array}$ & Missings & Mean [SD] & $\begin{array}{l}\text { Median } \\
{[\mathrm{IQR}]}\end{array}$ & Score range & $\min ^{\mathrm{b}}$ & $\max ^{b}$ & $\begin{array}{l}\text { Cronbach's } \\
\alpha^{\mathrm{c}}\end{array}$ \\
\hline $\begin{array}{l}\text { Physical } \\
\text { summary }^{\mathrm{a}}\end{array}$ & & $\begin{array}{l}\text { Lower summary } \\
\text { score represents } \\
\text { lower physical } \\
\text { health }^{\mathrm{a}}\end{array}$ & $\begin{array}{l}\text { Higher summary } \\
\text { score represents } \\
\text { higher physical } \\
\text { health }^{\mathrm{a}}\end{array}$ & 101 & $56.8[6.5]$ & $58.4[4.9]$ & $-8.6-67.2$ & na & na & $0.71^{\mathrm{d}}$ \\
\hline $\begin{array}{l}\text { Psychosocial } \\
\text { summary }^{\mathrm{a}}\end{array}$ & & $\begin{array}{l}\text { Lower summary } \\
\text { score represents } \\
\text { lower psychosocial } \\
\text { health }^{\mathrm{a}}\end{array}$ & $\begin{array}{l}\text { Higher summary } \\
\text { score represents } \\
\text { higher } \\
\text { psychosocial } \\
\text { health }^{\mathrm{a}}\end{array}$ & 101 & $53.0[6.6]$ & $53.8[8.1]$ & $12.2-66.2$ & na & na & $0.83^{\mathrm{d}}$ \\
\hline $\begin{array}{l}\text { Physical } \\
\text { functioning }\end{array}$ & 3 & $\begin{array}{l}\text { Child is limited a lot } \\
\text { in performing all } \\
\text { physical activities, } \\
\text { including self care, } \\
\text { because of health }\end{array}$ & $\begin{array}{l}\text { Child performs all } \\
\text { types of physical } \\
\text { activities, } \\
\text { including the most } \\
\text { vigorous, without } \\
\text { limitations } \\
\text { attributable to } \\
\text { health }\end{array}$ & 28 & $97.2[11.0]$ & $100.0[0.0]$ & $0-100$ & 0.2 & 90.6 & 0.86 \\
\hline $\begin{array}{l}\text { Role funct.- } \\
\text { emo/behav. }\end{array}$ & 1 & $\begin{array}{l}\text { Child is limited a lot } \\
\text { in school work or } \\
\text { activities with } \\
\text { friends as a result } \\
\text { of emotional or } \\
\text { behaviour } \\
\text { problems }\end{array}$ & $\begin{array}{l}\text { Child has no } \\
\text { limitations in } \\
\text { schoolwork or } \\
\text { activities with } \\
\text { friends as a result } \\
\text { of emotional or } \\
\text { behaviour } \\
\text { problems }\end{array}$ & 26 & 97.5 [10.9] & $100.0[0.0]$ & $0-100$ & 0.2 & 94.2 & na \\
\hline $\begin{array}{c}\text { Role funct.- } \\
\text { physical }\end{array}$ & 1 & $\begin{array}{l}\text { Child is limited a lot } \\
\text { in school work or } \\
\text { activities with } \\
\text { friends as a result } \\
\text { of physical health }\end{array}$ & $\begin{array}{l}\text { Child has no } \\
\text { limitations in } \\
\text { schoolwork or } \\
\text { activities with } \\
\text { friends as a result } \\
\text { of physical health }\end{array}$ & 27 & $97.0[11.9]$ & $100.0[0.0]$ & $0-100$ & 0.2 & 93.1 & na \\
\hline Bodily pain & 1 & $\begin{array}{l}\text { Child has extremely } \\
\text { severe, frequent, } \\
\text { and limiting bodily } \\
\text { pain }\end{array}$ & $\begin{array}{l}\text { Child has no pain or } \\
\text { limitations because } \\
\text { of pain }\end{array}$ & 28 & $85.7[16.1]$ & $80.0[20.0]$ & $0-100$ & 0.6 & 44.7 & na \\
\hline $\begin{array}{l}\text { General } \\
\text { behavior }\end{array}$ & 4 & $\begin{array}{l}\text { Child very often } \\
\text { exhibits } \\
\text { aggressive, } \\
\text { immature, } \\
\text { delinquent } \\
\text { behaviour }\end{array}$ & $\begin{array}{l}\text { Child never exhibits } \\
\text { aggressive, } \\
\text { immature, } \\
\text { delinquent } \\
\text { behaviour }\end{array}$ & 17 & $71.8[14.3]$ & $71.3[18.75]$ & $0-100$ & 0.0 & 2.6 & 0.69 \\
\hline Mental health & 3 & $\begin{array}{l}\text { Child has feelings of } \\
\text { anxiety and } \\
\text { depression all of } \\
\text { the time }\end{array}$ & $\begin{array}{l}\text { Child feels peaceful, } \\
\text { happy, and calm } \\
\text { all of the time }\end{array}$ & 28 & $81.7[14.2]$ & 83.3 [16.7] & $0-100$ & 0.0 & 20.5 & 0.64 \\
\hline Self-esteem & 3 & $\begin{array}{l}\text { Child is very } \\
\text { dissatisfied with } \\
\text { abilities, looks, } \\
\text { family/peer } \\
\text { relationships, and } \\
\text { life overall }\end{array}$ & $\begin{array}{l}\text { Child is very } \\
\text { satisfied with } \\
\text { abilities, looks, } \\
\text { family/peer } \\
\text { relationships' and } \\
\text { life overall }\end{array}$ & 22 & $82.1[13.1]$ & $75.0[16.7]$ & $0-100$ & 0.1 & 24.3 & 0.82 \\
\hline $\begin{array}{l}\text { Gen health } \\
\text { perc. }\end{array}$ & 4 & $\begin{array}{l}\text { Parent believes } \\
\text { child's health is } \\
\text { poor and likely to } \\
\text { get worse }\end{array}$ & $\begin{array}{l}\text { Parent believes } \\
\text { child's health is } \\
\text { excellent and will } \\
\text { continue to be so }\end{array}$ & 17 & $85.1[15.7]$ & $90.0[18.8]$ & $7.5-100$ & 0.0 & 21.7 & 0.49 \\
\hline $\begin{array}{l}\text { Parental- } \\
\text { emotional }\end{array}$ & 2 & $\begin{array}{l}\text { Parent experiences a } \\
\text { great deal of } \\
\text { emotional worry/ } \\
\text { concern as a result } \\
\text { of child's physical } \\
\text { and/or } \\
\text { psychosocial health }\end{array}$ & $\begin{array}{l}\text { Parent doesn't } \\
\text { experience feelings } \\
\text { of emotional } \\
\text { worry/concern as a } \\
\text { result of child's } \\
\text { physical and/or } \\
\text { psychosocial } \\
\text { health }\end{array}$ & 20 & $89.2[14.5]$ & $100.0[12.5]$ & $0-100$ & 0.1 & 51.3 & 0.38 \\
\hline
\end{tabular}


Table 1 continued

\begin{tabular}{|c|c|c|c|c|c|c|c|c|c|c|}
\hline \multicolumn{4}{|c|}{ CHQ-PF28 scales } & \multicolumn{7}{|c|}{ Overall $(n=3,227)$} \\
\hline & $\begin{array}{l}\text { Number } \\
\text { of items }\end{array}$ & $\begin{array}{l}\text { Description } \\
\text { low score }\end{array}$ & $\begin{array}{l}\text { Description } \\
\text { high score }\end{array}$ & Missings & Mean [SD] & $\begin{array}{l}\text { Median } \\
{[\mathrm{IQR}]}\end{array}$ & Score range & $\begin{array}{l}\% \\
\min ^{\mathrm{b}}\end{array}$ & $\max ^{b}$ & $\begin{array}{l}\text { Cronbach's } \\
\alpha^{\mathrm{c}}\end{array}$ \\
\hline Parental-time & 2 & $\begin{array}{l}\text { Parent experiences a } \\
\text { lot of limitations in } \\
\text { time available for } \\
\text { personal needs } \\
\text { because of child's } \\
\text { physical and/or } \\
\text { psychosocial health }\end{array}$ & $\begin{array}{l}\text { Parent doesn't } \\
\text { experience } \\
\text { limitations in time } \\
\text { available for } \\
\text { personal needs } \\
\text { because of child's } \\
\text { physical and/or } \\
\text { psychosocial } \\
\text { health }\end{array}$ & 27 & $95.2[12.8]$ & $100.0[0.0]$ & $0-100$ & 0.2 & 82.7 & 0.56 \\
\hline $\begin{array}{l}\text { Family } \\
\text { activities }\end{array}$ & 2 & $\begin{array}{l}\text { The child's health } \\
\text { very often limits } \\
\text { and interrupts } \\
\text { family activities or } \\
\text { is a source of } \\
\text { family tension }\end{array}$ & $\begin{array}{l}\text { The child's health } \\
\text { never limits or } \\
\text { interrupts family } \\
\text { activities or is a } \\
\text { source of family } \\
\text { tension }\end{array}$ & 24 & $89.2[16.3]$ & $100.0[25.0]$ & $0-100$ & 0.2 & 59.6 & 0.70 \\
\hline $\begin{array}{l}\text { Family } \\
\text { cohesion }\end{array}$ & 1 & $\begin{array}{l}\text { Family's ability to } \\
\text { get along is rated } \\
\text { "poor" }\end{array}$ & $\begin{array}{l}\text { Family's ability to } \\
\text { get along is rated } \\
\text { "excellent" }\end{array}$ & 63 & $73.6[17.8]$ & $85.0[25.0]$ & $0-100$ & 0.1 & 14.8 & na \\
\hline $\begin{array}{l}\text { Change in } \\
\text { health }\end{array}$ & 1 & $\begin{array}{l}\text { Child's health is } \\
\text { much worse now } \\
\text { than } 1 \text { year ago }\end{array}$ & $\begin{array}{l}\text { Child's health is } \\
\text { much better now } \\
\text { than } 1 \text { year ago }\end{array}$ & 34 & $56.2[15.4]$ & $50.0[0.0]$ & $0-100$ & 0.2 & 7.9 & na \\
\hline
\end{tabular}

Score interpretation: Reproduced with permission from the principal author Landgraf (page 38-39) [38]

na not applicable

a Physical and Psychosocial CHQ summary scores based on a factor-analytical model on U.S. population samples. A score of 50 represents the mean in the general U.S. population; scores above/below 50 are above/below the average in the general U.S. population [38]. Summary scales do not include the family activities, family cohesion and change in health scales

b $\%$ of respondent with the highest, respectively lowest possible CHQ-PF28 scale score (ceiling/floor)

C Average Cronbach's $\alpha$ of the eight multi-item scales 0.77

d Cronbach's $\alpha$ of the summary scales might be higher because the single-item scales (role func-emo/behav, role func-physical and bodily pain) could not be included

and less boys than girls in the overweight and obese subgroups (37.0 and $26 \%$, respectively). The mean age of the mother was 36.5 years [SD: 4.3], $89.3 \%$ of mothers were born in the Netherlands, and $93.5 \%$ were married or in a de facto relationship (Table 2).

In Table 3, the mean and median scores on the CHQPF28 scales are presented. Table 4 presents the bootstrap sampling results of the general linear models.

\section{CHQ-PF28 summary scales}

A significant lower parent-reported score on the physical summary scale for underweight children compared to normal weight children $(p<0.01)$ was found with the general linear model (adjusted model: $\beta,-1.11 ; 95 \% \mathrm{CI},-1.85$ to -0.39 , $p<0.01$ ). Additionally, severely obese children (adjusted model; $\beta-2.60,95 \% \mathrm{CI}-4.80$ to $-0.57, p<0.01$ ) showed lower parent-reported scores on the physical summary scale (Table 3). The general linear model showed no association with lower parent-reported scores on the physical summary scale for overweight and obese children compared to normal weight children $(p>0.05)$. There were no significant differences on the psychosocial summary scale scores between the different weight categories (Table 4).

\section{CHQ-PF28 profile scales}

Parents of overweight, obese and severely obese children reported statistically significant lower scores on the physical functioning scale $(p<0.05, p<0.001$ and $p<0.001$ respectively) compared to parents of normal weight children (Table 3); for overweight and severely obese children, this was also found with the general linear model (Table 4).

Parents of obese children reported significant lower scores on self-esteem scales $(p<0.05$, adjusted model beta, $-4.18 ; 95 \%$ CI, -7.87 to -0.78 ) (Table 4). Parents of severely obese children reported lower scores regarding the parental impact-emotional and parental impact-time scale $(p<0.05)$ (Table 3).

Obese and underweight children both had lower parentreported scores on the general health perception scale ( $p<0.05$ and $p<0.01$ respectively) (Table 3 ). For underweight children, an association with lower parent-reported scores on the bodily pain (adjusted model: $\beta-2.42,95 \%$ CI: 
Table 2 General characteristics of the study population, stratified by children's weight status $(n=3,227)$

\begin{tabular}{|c|c|c|c|c|c|c|}
\hline & $\begin{array}{l}\text { Total } \\
(n=3,227)\end{array}$ & $\begin{array}{l}\text { Underweight }^{\mathrm{a}} \\
(n=449)\end{array}$ & $\begin{array}{l}\text { Normal } \\
\text { weight }^{\text {a }} \\
(n=2,278)\end{array}$ & $\begin{array}{l}\text { Overweight }^{\mathrm{a}} \\
(n=405)\end{array}$ & $\begin{array}{l}\text { Obesity }^{\mathrm{a}} \\
(n=50)\end{array}$ & $\begin{array}{l}\text { Severe } \\
\text { obesity }^{\mathrm{a}} \\
(n=45)\end{array}$ \\
\hline \multicolumn{7}{|l|}{ Child characteristics } \\
\hline $\begin{array}{l}\text { Age in years, mean } \pm \text { SD } \\
\text { Gender** }\end{array}$ & $5.7 \pm 0.4$ & $5.8 \pm 0.4$ & $5.7 \pm 0.4$ & $5.8 \pm 0.4$ & $5.8 \pm 0.4$ & $5.9 \pm 0.4$ \\
\hline$\%$ boys & 49.3 & 50.8 & 51.4 & 37.0 & 26.0 & 66.7 \\
\hline \multicolumn{7}{|l|}{ Ethnicity (34 missings) } \\
\hline$\%$ Dutch & 98.3 & 97.3 & 98.4 & 99.0 & 95.9 & 97.7 \\
\hline $\mathrm{BMI}^{* *}$, mean $\pm \mathrm{SD}$ & $15.7 \pm 1.7$ & $13.4 \pm 0.5$ & $15.45 \pm 0.9$ & $18.2 \pm 0.6$ & $20.2 \pm 0.4$ & $21.7 \pm 1.1$ \\
\hline $\begin{array}{l}\text { Number of chronic } \\
\text { conditions, } \\
\text { mean } \pm \text { SD }\end{array}$ & $0.6 \pm 0.9$ & $0.6 \pm 0.8$ & $0.5 \pm 0.9$ & $0.6 \pm 0.9$ & $0.7 \pm 1.2$ & $0.6 \pm 0.8$ \\
\hline \multicolumn{7}{|l|}{ Number of chronic conditions } \\
\hline$\%$ no chronic condition & 62.4 & 60.9 & 62.9 & 61.5 & 68.0 & 57.8 \\
\hline \multicolumn{7}{|l|}{ Characteristics of the mother } \\
\hline $\begin{array}{l}\text { Age in years, mean } \pm \mathrm{SD} \\
(n=38 \text { missings })\end{array}$ & $36.5 \pm 4.3$ & $36.7 \pm 4.0$ & $36.5 \pm 4.3$ & $35.3 \pm 3.9$ & $36.0 \pm 5.2$ & $36.1 \pm 6.0$ \\
\hline \multicolumn{7}{|l|}{ Gender ( $n=32$ missings $)$} \\
\hline$\%$ mother is respondent & 89.3 & 88.5 & 89.5 & 89.3 & 83.7 & 93.2 \\
\hline \multicolumn{7}{|l|}{$\begin{array}{l}\text { Born in the Netherlands*** } \\
(n=32 \text { missings })\end{array}$} \\
\hline$\%$ yes & 90.9 & 91.0 & 92.0 & 87.4 & 79.6 & 77.3 \\
\hline \multicolumn{7}{|l|}{$\begin{array}{l}\text { Educational level }{ }^{* * *} \\
(n=49 \text { missings })\end{array}$} \\
\hline$\%$ low & 3.1 & 2.3 & 2.6 & 4.8 & 8.2 & 14.3 \\
\hline$\%$ mid-low & 17.7 & 14.9 & 16.8 & 23.1 & 26.5 & 35.7 \\
\hline$\%$ mid-high & 46.1 & 46.8 & 46.1 & 47.0 & 44.9 & 31.0 \\
\hline$\%$ high & 33.1 & 36.0 & 34.4 & 25.1 & 20.4 & 19.0 \\
\hline \multicolumn{7}{|l|}{$\begin{array}{l}\text { Marital status } * * * \\
(n=46 \text { missings })\end{array}$} \\
\hline $\begin{array}{l}\% \text { married or living together } \\
\text { Weight status*** } \\
(n=93 \text { missings })\end{array}$ & 93.5 & 92.7 & 94.5 & 92.1 & 85.3 & 80.5 \\
\hline$\%$ normal weight & 70.4 & 81.5 & 72.5 & 52.7 & 47.5 & 41.0 \\
\hline
\end{tabular}

$* p<0.05 ; * p<0.01 ; * * * p<0.001: p$ value from chi-square tests for categorical variables and ANOVA for continues variables comparing general characteristics across weight categories

${ }^{\text {a }}$ Categories based on international age- and gender-specific BMI cutoff values, for severe obesity cutoff values of BMI: 20.00 in boys and BMI: 21.00 in girls were used \pm . According to the World Health Organization definition, BMI < 25: normal weight, BMI 25-30: overweight, BMI > 30: obesity [41]

-4.28 to $-0.65, p<0.01)$ was found compared to normal weight children (Table 4).

\section{Effect modification}

The physical and psychosocial summary scale showed a significant interaction between weight and child ethnicity $(p<0.05)$; Dutch children showed on average lower parent-reported scores than the non-Dutch children across all weight categories. In similar direction, we found an interaction between weight and child ethnicity $(p<0.05)$ for the physical functioning and the self-esteem scale.
Additionally, a significant interaction was found between weight and gender for the scale of general health $(p<0.05)$; parent-reported scores for boys were on average lower than for girls across weight categories.

\section{Discussion}

Our findings showed lower parent-reported physical healthrelated quality of life scores of severely obese and underweight children entering elementary school compared to normal weight children. Parent-reported psychosocial health-related 


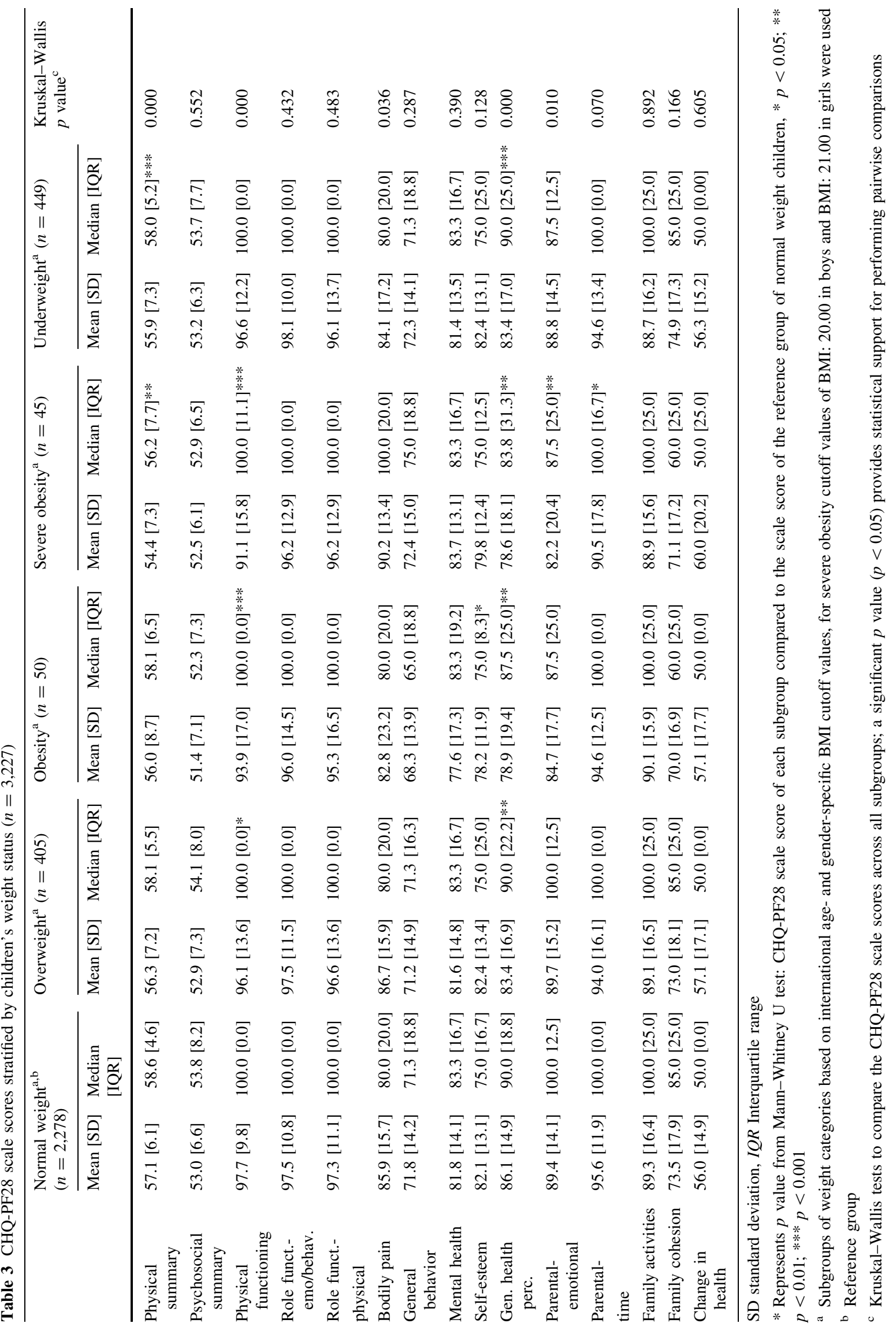


Table 4 Associations between children's weight status and CHQ-PF28 scale scores $(n=3,227)$

\begin{tabular}{|c|c|c|c|c|c|c|c|c|}
\hline & Overweight $^{\mathrm{a}}(n=405)$ & $p$ value & Obesity $^{\mathrm{a}}(n=50)$ & $p$ value & $\begin{array}{l}\text { Severe obesity }{ }^{\mathrm{a}} \\
(n=45)\end{array}$ & $p$ value & $\begin{array}{l}\text { Underweight }^{\mathrm{a}} \\
(n=449)\end{array}$ & $p$ value \\
\hline \multicolumn{9}{|l|}{ Physical summary } \\
\hline $\begin{array}{l}\text { Bootstrap } \\
\text { unadjusted } * *\end{array}$ & $-0.80(-1.61 ;-0.06)$ & 0.038 & $-1.04(-3.79 ; 1.27)$ & 0.424 & $-2.72(-5.14 ;-0.65)$ & 0.008 & $-1.15(--.95 ;-0.42)$ & 0.000 \\
\hline $\begin{array}{l}\text { Bootstrap } \\
\text { adjusted } * *\end{array}$ & $-0.59(-1.37 ; 0.17)$ & 0.138 & $-1.12(-3.56 ; 1.27)$ & 0.380 & $-2.60(-4.80 ;-0.57)$ & 0.006 & $-1.11(-1.85 ;-0.39)$ & 0.002 \\
\hline \multicolumn{9}{|l|}{ Psychosocial summary } \\
\hline $\begin{array}{l}\text { Bootstrap } \\
\text { unadjusted }\end{array}$ & $-0.15(-0.92 ; 0.66)$ & 0.692 & $-1.70(-3.89 ; 0.30)$ & 0.114 & $-0.57(-2.43 ; 1.27)$ & 0.564 & $0.22(-0.45 ; 0.89)$ & 0.506 \\
\hline Bootstrap adjusted & $-0.13(-0.96 ; 0.69)$ & 0.734 & $-1.39(-4.00 ; 0.91)$ & 0.280 & $-0.28(-2.32 ; 1.77)$ & 0.794 & $0.27(-0.43 ; 0.95)$ & 0.454 \\
\hline \multicolumn{9}{|l|}{ Physical functioning } \\
\hline $\begin{array}{l}\text { Bootstrap } \\
\text { unadjusted }\end{array}$ & $-1.66(-3.12 ;-0.29)$ & 0.014 & $-3.95(-9.40 ; 0.16)$ & 0.062 & $-6.71(-11.47 ;-2.25)$ & 0.000 & $-1.16(-2.40 ; 0.06)$ & 0.058 \\
\hline $\begin{array}{l}\text { Bootstrap } \\
\text { adjusted }^{* *}\end{array}$ & $-1.56(-3.04 ;-0.08)$ & 0.040 & $-3.78(-9.57 ; 0.96)$ & 0.124 & $-5.93(-10.32 ;-1.94)$ & 0.002 & $-1.00(-2.23 ; 0.08)$ & 0.060 \\
\hline \multicolumn{9}{|l|}{ Role funct.-emo/behav. } \\
\hline $\begin{array}{l}\text { Bootstrap } \\
\text { unadjusted }\end{array}$ & $0.03(-1.19 ; 1.13)$ & 0.930 & $-1.46(-6.00 ; 1.98)$ & 0.512 & $-1.34(-5.88 ; 1.98)$ & 0.526 & $0.59(-0.47 ; 1.54)$ & 0.268 \\
\hline Bootstrap adjusted & $0.07(-1.40 ; 1.38)$ & 0.874 & $-1.37(-7.05 ; 2.95)$ & 0.624 & $-0.49(-4.96 ; 3.02)$ & 0.862 & $0.64(-0.38 ; 1.65)$ & 0.246 \\
\hline \multicolumn{9}{|l|}{ Role funct.-physical } \\
\hline $\begin{array}{l}\text { Bootstrap } \\
\text { unadjusted }\end{array}$ & $-0.69(-2.21 ; 0.71)$ & 0.340 & $-2.00(-6.66 ; 1.82)$ & 0.390 & $-1.19(-5.20 ; 2.15)$ & 0.572 & $-1.15(-2.52 ; 0.17)$ & 0.088 \\
\hline Bootstrap adjusted & $-0.95(-2.82 ; 0.65)$ & 0.234 & $-2.32(-7.81 ; 2.15)$ & 0.396 & $-1.38(-5.75 ; 2.28)$ & 0.544 & $-1.18(-2.65 ; 0.14)$ & 0.082 \\
\hline \multicolumn{9}{|l|}{ Bodily pain } \\
\hline $\begin{array}{l}\text { Bootstrap } \\
\text { unadjusted* }\end{array}$ & $0.85(-0.89 ; 2.40)$ & 0.320 & $-3.19(-10.25 ; 2.87)$ & 0.344 & $4.27(0.09 ; 8.23)$ & 0.048 & $-1.80(-3.68 ;-0.09)$ & 0.034 \\
\hline $\begin{array}{l}\text { Bootstrap } \\
\text { adjusted* }\end{array}$ & $0.84(-0.93 ; 2.53)$ & 0.340 & $-3.15(-10.58 ; 3.16)$ & 0.360 & $1.85(-2.52 ; 5.88)$ & 0.400 & $-2.42(-4.28 ;-0.65)$ & 0.008 \\
\hline \multicolumn{9}{|l|}{ General behavior } \\
\hline $\begin{array}{l}\text { Bootstrap } \\
\text { unadjusted }\end{array}$ & $-0.66(-2.23 ; 0.76)$ & 0.382 & $-3.60(-7.51 ; 0.40)$ & 0.080 & $0.48(-4.01 ; 4.66)$ & 0.808 & $0.48(-0.95 ; 1.87)$ & 0.520 \\
\hline Bootstrap adjusted & $-0.56(-2.29 ; 1.18)$ & 0.526 & $-3.28(7.71 ; 1.22)$ & 0.156 & $1.19(-3.94 ; 5.81)$ & 0.632 & $0.28(-1.29 ; 1.88)$ & 0.718 \\
\hline \multicolumn{9}{|l|}{ Mental health } \\
\hline $\begin{array}{l}\text { Bootstrap } \\
\text { unadjusted }\end{array}$ & $-0.24(-1.75 ; 1.29)$ & 0.790 & $-4.30(-9.29 ; 0.43)$ & 0.076 & $2.00(-2.09 ; 5.69)$ & 0.324 & $-0.48(-1.94 ; 0.91)$ & 0.488 \\
\hline Bootstrap adjusted & $-0.27(-1.93 ; 1.45)$ & 0.782 & $-2.20(-7.23 ; 2.81)$ & 0.408 & $2.39(-2.04 ; 6.30)$ & 0.282 & $-0.03(-1.52 ; 1.44)$ & 1.000 \\
\hline \multicolumn{9}{|l|}{ Self-esteem } \\
\hline $\begin{array}{l}\text { Bootstrap } \\
\text { unadjusted }\end{array}$ & $0.29(-1.09 ; 1.59)$ & 0.684 & $-3.93(-7.37 ;-0.35)$ & 0.026 & $-2.26(-5.91 ; 1.25)$ & 0.228 & $0.28(-1.09 ; 1.58)$ & 0.690 \\
\hline Bootstrap adjusted & $0.25(-1.23 ; 1.69)$ & 0.736 & $-4.18(-7.87 ;-0.78)$ & 0.012 & $-1.25(-4.96 ; 2.59)$ & 0.512 & $0.27(-1.29 ; 1.67)$ & 0.712 \\
\hline \multicolumn{9}{|l|}{ Gen. health perc. } \\
\hline $\begin{array}{l}\text { Bootstrap } \\
\text { unadjusted } * * *\end{array}$ & $-2.68(-4.50 ;-0.90)$ & 0.002 & $-7.06(-12.55 ;-1.89)$ & 0.006 & $-7.65(-12.96 ;-2.58)$ & 0.000 & $-2.62(-4.16 ;-1.07)$ & 0.000 \\
\hline $\begin{array}{l}\text { Bootstrap } \\
\text { adjusted } * *\end{array}$ & $-1.90(-3.67 ; 0.005)$ & 0.052 & $-5.39(-10.33 ;-0.78)$ & 0.018 & $-4.41(-9.91 ; 0.79)$ & 0.090 & $-2.07(-3.77 ;-0.45)$ & 0.008 \\
\hline \multicolumn{9}{|l|}{ Parental-emotional } \\
\hline $\begin{array}{l}\text { Bootstrap } \\
\text { unadjusted } *\end{array}$ & $0.32(-1.24 ; 1.89)$ & 0.670 & $-4.81(-10.01 ; 0.20)$ & 0.054 & $-7.41(-13.79 ;-1.75)$ & 0.008 & $-0.68(-2.19 ; 0.74)$ & 0.378 \\
\hline Bootstrap adjusted & $-0.18(-1.96 ; 1.60)$ & 0.852 & $-4.55(-10.43 ; 0.71)$ & 0.094 & $-6.77(-13.45 ;-0.69)$ & 0.020 & $-0.60(-2.15 ; 0.89)$ & 0.450 \\
\hline \multicolumn{9}{|l|}{ Parental-time } \\
\hline $\begin{array}{l}\text { Bootstrap } \\
\text { unadjusted }\end{array}$ & $-1.65(-3.47 ;-0.08)$ & 0.042 & $-1.04(-4.55 ; 1.92)$ & 0.610 & $-5.09(-10.87 ;-0.34)$ & 0.034 & $-1.00(-2.34 ; 0.34)$ & 0.160 \\
\hline Bootstrap adjusted & $-0.53(-2.22 ; 1.04)$ & 0.548 & $-0.06(-4.08 ; 3.32)$ & 0.980 & $-5.35(-11.92 ;-0.24)$ & 0.046 & $-1.41(-2.91 ; 0.07)$ & 0.060 \\
\hline \multicolumn{9}{|l|}{ Family activities } \\
\hline $\begin{array}{l}\text { Bootstrap } \\
\text { unadjusted }\end{array}$ & $-0.13(-1.90 ; 1.68)$ & 0.870 & $0.82(-3.72 ; 4.93)$ & 0.728 & $-0.43(-5.20 ; 4.02)$ & 0.894 & $-0.49(-2.17 ; 1.17)$ & 0.572 \\
\hline Bootstrap adjusted & $-0.004(-1.86 ; 1.98)$ & 0.996 & $1.90(-3.08 ; 6.61)$ & 0.434 & $0.85(-4.80 ; 5.55)$ & 0.736 & $-0.44(-2.22 ; 1.26)$ & 0.628 \\
\hline
\end{tabular}


Table 4 continued

\begin{tabular}{|c|c|c|c|c|c|c|c|c|}
\hline & Overweight $^{\mathrm{a}}(n=405)$ & $p$ value & Obesity $^{\mathrm{a}}(n=50)$ & $p$ value & $\begin{array}{l}\text { Severe obesity } \\
(n=45)\end{array}$ & $p$ value & $\begin{array}{l}\text { Underweight }^{\mathrm{a}} \\
(n=449)\end{array}$ & $p$ value \\
\hline \multicolumn{9}{|l|}{ Family cohesion } \\
\hline $\begin{array}{l}\text { Bootstrap } \\
\text { unadjusted }\end{array}$ & $-0.55(-2.60 ; 1.54)$ & 0.564 & $-3.45(-8.01 ; 0.88)$ & 0.134 & $-2.62(-7.30 ; 2.67)$ & 0.316 & $1.41(-0.36 ; 3.16)$ & 0.116 \\
\hline Bootstrap adjusted & $0.07(-2.08 ; 2.13)$ & 0.962 & $-4.43(-9.31 ; 0.51)$ & 0.082 & $-1.56(-7.34 ; 4.91)$ & 0.594 & $0.74(-1.31 ; 2.78)$ & 0.504 \\
\hline \multicolumn{9}{|l|}{ Change in health } \\
\hline $\begin{array}{l}\text { Bootstrap } \\
\text { unadjusted }\end{array}$ & $1.08(-0.57 ; 2.91)$ & 0.214 & $1.20(-3.38 ; 6.38)$ & 0.652 & $3.89(-1.76 ; 10.20)$ & 0.202 & $0.28(-1.15 ; 1.80)$ & 0.726 \\
\hline Bootstrap adjusted & $0.43(-1.30 ; 2.35)$ & 0.642 & $-0.06(-5.18 ; 5.49)$ & 0.970 & $0.86(-4.73 ; 7.21)$ & 0.814 & $0.15(1.44 ; 1.71)$ & 0.832 \\
\hline
\end{tabular}

Bootstrap analyses were performed for the general linear model; values are beta coefficients relative to the normal weight reference group with $95 \%$ confidence intervals. Numbers in boldface indicate a significant beta-coefficient. Unadjusted bootstrap: weight category as independent variable and the CHQ-PF28 scale as dependent variables, no correction for potential confounding variables. Adjusted bootstrap: the model corrected for potential confounding variables; weight category, child characteristics (gender, age, ethnicity, number of chronic conditions) and maternal characteristics (age, country of birth, education level, marital status and overweight yes/no) as independent variables and the CHQ-PF28 scales as dependent variables

* Represents significance level of weight category in the overall bootstrapped model, $* p<0.05 ; * * p<0.01 ; * * *<<0.001$

${ }^{\text {a }}$ Subgroups of weight categories based on international age- and gender-specific BMI cutoff values, for severe obesity cutoff values of BMI: 20.00 in boys and BMI: 21.00 in girls were used

quality of life scores were not significantly different across weight categories in the present study.

Overweight, obesity and health-related quality of life

After adjusting for potential confounding factors such as child gender and child age, the CHQ parent-reported physical summary score was lower among severely obese children. These findings of 5-6-year-old children are in line with findings among adolescents and older school-aged children [9, 11, 14, 15, 19, 20, 27, 45, 46].

The mechanisms underlying the association between BMI and health-related quality of life are not clear yet. It is possible that parents suspect that their overweight children are not able to join their peers in the same level of physical activity, for example during playtime, which thus may contribute to low scores for overweight children on the physical summary scales in our study. It has been hypothesized that physical activity has a BMI-independent positive effect on health-related quality of life [19, 47]. Shoup et al. [10] reported that compared to overweight children who did not meet the recommended physical activity guidelines, older overweight children reported better overall health-related quality of life when they met the recommended physical activity guidelines. Additional analyses (data not shown) in which we studied the association between health-related quality of life and playing outside showed that the amount of outside play (more than $1 \mathrm{~h}$ per day) was associated with higher scores on healthrelated quality of life $(p<0.01)$. However, the association between weight status and health-related quality of life remained statistically significant after adjustment for the amount of outside play (data not shown). So, parents of overweight and obese children report lower health-related quality of life, independent of the level of physical activity of their children.

Our finding that the presence of overweight and obesity in 5-6-year-old children is not associated with the psychosocial summary scale of health-related quality of life-is comparable with the findings reported by others [18, 20, 48]. Decrements to the psychosocial dimension of healthrelated quality of life seem to become more pronounced during adolescence as young teens become more aware of their physical appearance [25]. Additionally, the use of parent reports might influence the results on psychosocial health-related quality of life in our study. Literature suggests that only few parents realize that their child is overweight or obese at this age [49]; perhaps, parents are therefore not keen on problems that may impact their child's psychosocial health. On the other hand, parents might ignore psychosocial problems or not take them seriously because they suppose the child is not old enough to be unhappy due to his or her weight.

When exploring the results on the profile scales, it is noteworthy that the parental impact-emotional and parental impact-time scales had significantly lower parent-reported scores for severely obese children. Lower scores can be interpreted as parents having more concerns and less personal time due to their child's weight, than do parents of normal weight children. This finding has been reported by Wake et al. [20].

Underweight and health-related quality of life

Parents of underweight children reported lower physical health-related quality of life as compared to parents of normal weight children. These findings are in accordance with previously reported findings among older underweight 
children and adolescents [20, 24, 27]. Additionally, although analyses were exploratory, significantly more impairment was reported by parents on the bodily pain scale for underweight children. This has been reported only for boys in the study of Wake et al. [20].

We have no explanation for the low health-related quality of life scores reported by parents on the physical domain for underweight young children. In our study, there were no differences in the number of chronic health conditions experienced by underweight children. We have not measured short-term infections such as having the flu or a cold, which might have more impact or influence the amount of energy and physical functioning in underweight children.

There were no impairments reported by the parents of underweight children on the psychosocial dimension, contrary to what has been reported among adolescents [50, 51]. Lower school functioning scores [25] and lower self-esteem scores [11] have been reported among underweight children. We hypothesize that the psychosocial domains of health-related quality of life in young children with overweight may be unaffected or that parents do not recognize impairments on this domain. The impact of lower psychosocial health-related quality of life may have more consequences when children enter early and/or late adolescence.

Effect modification in the association between healthrelated quality of life and weight status

On average, parents reported children of "Dutch" origin, relative to "non-Dutch" children, to have lower scores on both the physical and psychosocial summary scales and on the physical functioning and self-esteem scales across all weight categories. The findings for the lower physical scale scores are consistent with previous studies documenting differences among white adolescents and adolescents of African heritage [50]. In the current study, difference in culture between Dutch and non-Dutch parents may have resulted in different reporting. Future research specifying ethnic subgroups instead of a broad ethnic group will have to provide more insight into the relation between ethnicity, weight status and health-related quality of life.

Significantly lower parent-reported scores for boys compared to girls regarding the general health scale were found across all weight categories. Griffiths [18] emphasized gender differences on psychosocial measures of health-related quality of life. However, this review was primarily based on studies among older children. In our study, the use of parent report in combination with the young age of the children might have contributed to a more equal scoring for both genders on the psychosocial and physical health-related quality of life summary scales across all weight categories.
Strengths and limitations

Some methodological issues are worth noting. Strength of the current study is the large population-based sample available to investigate and compare health-related quality of life of overweight, obese, severely obese and underweight children with normal weight children.

Limitations of the current study include the cross-sectional data that were used to investigate the relationship between weight status and health-related quality of life, and longitudinal research is needed to make any assumptions regarding the causality of the associations found. The choice of confounding variables corrected for in the general linear models was based on the preexisting knowledge about social and biological determinants of weight. Not all potential confounders were measured; for example, no assessment regarding specific conditions or diseases, for example, Coeliac disease [52], was available. The number of common chronic conditions was included as potential confounder. The average number of common chronic conditions was not significantly different between weight categories and was no effect modifier in the association between weight status and health-related quality of life.

The parent form of the CHQ was a feasible measure within this large population-based study. However, the use of parent-proxy reports on a child's health-related quality of life should be taken into account when interpreting the findings $[45,51,53,54]$.

\section{Conclusions}

This study highlights that as early as elementary school, parents report lower physical health-related quality of life of underweight and severely obese children. Although the decreases in health-related quality of life at this young age are relatively small, they might indicate more decreases in health-related quality of life when children are older. As such, it suggests that nutrition, health education and other interventions must be focused on both parents and children at early ages. Longitudinal studies to evaluate natural and intervention induced developments in body weight and BMI, and the associations with healthrelated quality of life in childhood are recommended. In the meantime, we recommend physicians, parents and teachers to be aware of the potential negative impact on health-related quality of life in both overweight and underweight children.

Acknowledgments This study is funded by a grant from ZonMw, the Netherlands Organization for Health Research and Development (project no. 50-50110-98-355). 
Open Access This article is distributed under the terms of the Creative Commons Attribution License which permits any use, distribution, and reproduction in any medium, provided the original author(s) and the source are credited.

\section{References}

1. de Onis, M., Blossner, M., \& Borghi, E. (2010). Global prevalence and trends of overweight and obesity among preschool children. American Journal of Clinical Nutrition, 92(5), 1257-1264.

2. Schonbeck, Y., Talma, H., van Dommelen, P., Bakker, B., Buitendijk, S. E., Hirasing, R. A., et al. (2011). Increase in prevalence of overweight in Dutch children and adolescents: A comparison of nationwide growth studies in 1980, 1997 and 2009. PLoS ONE, 6(11), e27608.

3. Falkner, N. H., Neumark-Sztainer, D., Story, M., Jeffery, R. W., Beuhring, T., \& Resnick, M. D. (2001). Social, educational, and psychological correlates of weight status in adolescents. Obesity Research, 9(1), 32-42.

4. Reilly, J. J., \& Kelly, J. (2010). Long-term impact of overweight and obesity in childhood and adolescence on morbidity and premature mortality in adulthood: Systematic review. International Journal of Obesity (London).

5. Soltoft, F., Hammer, M., \& Kragh, N. (2009). The association of body mass index and health-related quality of life in the general population: Data from the 2003 Health Survey of England. Quality of Life Research, 18(10), 1293-1299.

6. Fontaine, K. R., \& Barofsky, I. (2001). Obesity and health-related quality of life. Obesity Reviews, 2, 173-182.

7. Boyle, S. E., Jones, G. L., \& Walters, S. J. (2010). Physical activity, quality of life, weight status and diet in adolescents. Quality of Life Research, 19, 943-954.

8. Modi, A. C., Loux, T. J., Bell, S. K., Harmon, C. M., Inge, T. H., \& Zeller, M. H. (2008). Weight-specific health-related quality of life in adolescents with extreme obesity. Obesity (Silver Spring), 16(10), 2266-2271.

9. Ottova, V., Erhart, M., Rajmil, L., Dettenborn-Betz, L., \& RavensSieberer, U. (2011). Overweight and its impact on the healthrelated quality of life in children and adolescents: Results from the European KIDSCREEN survey. Quality of Life Research.

10. Shoup, J. A., Gattshall, M., Dandamudi, P., \& Estabrooks, P. (2008). Physical activity, quality of life, and weight status in overweight children. Quality of Life Research, 17(3), 407-412.

11. Friedlander, S. L., Larkin, E. K., Rosen, C. L., Palermo, T. M., \& Redline, S. (2003). Decreased quality of life associated with obesity in school-aged children. Archives of Pediatrics and Adolescent Medicine, 157(12), 1206-1211.

12. Wille, N., Erhart, M., Petersen, C., \& Ravens-Sieberer, U. (2008). The impact of overweight and obesity on health-related quality of life in childhood-results from an intervention study. BMC Public Health, 8, 421.

13. Williams, J., Wake, M., Hesketh, K., Maher, E., \& Waters, E. (2005). Health-related quality of life of overweight and obese children. JAMA, 293(1), 70-76.

14. Williams, J. W., Canterford, L., Hesketh, K. D., Hardy, P., Waters, E. B., Patton, G. C., et al. (2011). Changes in body mass index and health related quality of life from childhood to adolescence. International Journal of Pediatric Obesity, 6(2-2), e442-e448.

15. Pinhas-Hamiel, O., Singer, S., Pilpel, N., Fradkin, A., Modan, D., \& Reichman, B. (2006). Health-related quality of life among children and adolescents: Associations with obesity. International Journal of Obesity (London), 30(2), 267-272.

16. de Beer, M., Hofsteenge, G. H., Koot, H. M., Hirasing, R. A., Delemarre-van de Waal, H. A., \& Gemke, R. J. (2007). Healthrelated-quality-of-life in obese adolescents is decreased and inversely related to BMI. Acta Paediatrica, 96(5), 710-714.

17. Gibson, L. Y., Byrne, S. M., Blair, E., Davis, E. A., Jacoby, P., \& Zubrick, S. R. (2008). Clustering of psychosocial symptoms in overweight children. Australian and New Zealand Journal of Psychiatry, 42(2), 118-125.

18. Griffiths, L. J., Parsons, T. J., \& Hill, A. J. (2010). Self-esteem and quality of life in obese children and adolescents: A systematic review. International Journal of Pediatric Obesity, 5(4), 282-304.

19. Tsiros, M. D., Olds, T., Buckley, J. D., Grimshaw, P., Brennan, L., Walkley, J., et al. (2009). Health-related quality of life in obese children and adolescents. International Journal of Obesity (London), 33, 387-400.

20. Wake, M., Salmon, L., Waters, E., Wright, M., \& Hesketh, K. (2002). Parent-reported health status of overweight and obese Australian primary school children: A cross-sectional population survey. International Journal of Obesity and Related Metabolic Disorders, 26(5), 717-724.

21. Cockrell Skinner, A., Perrin, E. M., \& Steiner, M. J. (2010). Healthy for now? A cross-sectional study of the comorbidities in obese preschool children in the United States. Clinical pediatrics (Phila), 49(7), 648-655.

22. van Buuren, S. (2004). Afkapwaarden van de 'body-mass index' (BMI) voor ondergewicht van Nederlandse kinderen. Nederlands Tijdschrift voor Geneeskunde, 148(40).

23. Garner, R. E., Feeny, D. H., Thompson, A., Bernier, J., McFarland, B. H., \& Huguet, N. et al. (2011). Bodyweight, gender, and quality of life: a population-based longitudinal study. Quality of Life Research (Epub ahead of print).

24. Swallen, K. C., Reither, E. N., Haas, S. A., \& Meier, A. M. (2005). Overweight, obesity, and health-related quality of life among adolescents: The National Longitudinal Study of Adolescent Health. Pediatrics, 115(2), 340-347.

25. Sato, H., Nakamura, N., \& Sasaki, N. (2008). Effects of bodyweight on health-related quality of life in school-aged children and adolescents. Pediatrics International, 50(4), 552-556.

26. Arif, A. A., \& Rohrer, J. E. (2006). The relationship between obesity, hyperglycemia symptoms, and health-related quality of life among Hispanic and non-Hispanic white children and adolescents. BMC Family Practice, 7, 3.

27. Ostbye, T., Malhotra, R., Wong, H. B., Tan, S. B., \& Saw, S. M. (2010). The effect of body mass on health-related quality of life among Singaporean adolescents: Results from the SCORM study. Quality of Life Research, 19(2), 167-176.

28. Liberty, K. A., Pattemore, P., Reid, J., \& Tarren-Sweeney, M. (2010). Beginning school with asthma independently predicts low achievement in a prospective cohort of children. Chest, 138(6), 1349-1355.

29. Sigfusdottir, I. D., Kristjansson, A. L., \& Allegrante, J. P. (2007). Health behaviour and academic achievement in Icelandic school children. Health Education Research, 22(1), 70-80.

30. Krukowski, R. A., West, D. S., Philyaw Perez, A., Bursac, Z., Phillips, M. M., \& Raczynski, J. M. (2009). Overweight children, weight-based teasing and academic performance. International Journal of Pediatric Obesity, 4(4), 274-280.

31. Raat, H., Mohangoo, A. D., \& Grootenhuis, M. A. (2006). Pediatric health-related quality of life questionnaires in clinical trials. Current opinion in Allergy and Clinical Immunology, 6(3), 180-185.

32. Raat, H., Botterweck, A. M., Landgraf, J. M., Hoogeveen, W. C., \& Essink-Bot, M. L. (2005). Reliability and validity of the short 
form of the child health questionnaire for parents (CHQ-PF28) in large random school based and general population samples. Journal of Epidemiology and Community Health, 59(1), 75-82.

33. Veldhuis, L., Struijk, M. K., Kroeze, W., Oenema, A., Renders, C. M., Bulk-Bunschoten, A. M., et al. (2009). 'Be active, eat right', evaluation of an overweight prevention protocol among 5-year-old children: Design of a cluster randomised controlled trial. BMC Public Health, 9, 177.

34. Bulk-Bunschoten, A. M. W., Renders, C. M., Van Leerdam, F. J. M., \& HiraSing, R. A. (2005). Youth health care overweightdetection-protocol. Woerden: Platform Jeugdgezondheidszorg.

35. Cole, T. J., Bellizzi, M. C., Flegal, K. M., \& Dietz, W. H. (2000). Establishing a standard definition for child overweight and obesity worldwide: International survey. BMJ, 320(7244), 1240-1243.

36. Cole, T. J., Flegal, K. M., Nicholls, D., \& Jackson, A. A. (2007). Body mass index cut offs to define thinness in children and adolescents: International survey. BMJ, 335(7612), 194.

37. Barlow, S. E., \& Expert Committee. (2007). Expert committee recommendations regarding the prevention, assessment, and treatment of child and adolescent overweight and obesity: Summary report. Pediatrics, 120(Suppl 4), S164-S192.

38. Landgraf, J. M., Abetz, L., \& Ware, J. E. (1996). The CHQ's user manual. Boston: The Health Institute, New England Medical Center.

39. Bender, R., \& Lange, S. (2001). Adjusting for multiple testingwhen and how? Journal of Clinical Epidemiology, 54(4), 343349.

40. Swertz, O., Duimelaar, P., \& Thijssen, J. (2004). Statistics Netherlands. Migrants in the Netherlands 2004. Voorburg/Heerlen, Netherlands: Statistics Netherlands.

41. World Health Organisation. (2011). Fact sheet nr 311: Obesity and overweight. http://www.who.int/mediacentre/factsheets/fs 311/en/index.html. Accessed 07 September 2011.

42. Statistics Netherlands. (2004). Dutch standard classification of education 2003. Voorburg/Heerlen, Netherlands: Statistics Netherlands.

43. Davison, A. C., \& Hinkley, D. V. (1997). Bootstrap methods and their applications. Cambridge: Cambridge University Press.

44. Walters, S. J., \& Campbell, M. J. (2005). The use of bootstrap methods for estimating sample size and analysing health-related quality of life outcomes. Statistics in Medicine, 24(7), 10751102.

45. Hughes, A. R., Stewart, L., Chapple, J., McColl, J. H., Donaldson, M. D., Kelnar, C. J., et al. (2008). Randomized, controlled trial of a best-practice individualized behavioral program for treatment of childhood overweight: Scottish Childhood Overweight Treatment Trial (SCOTT). Pediatrics, 121(3), e539-e546.

46. Zhang, L., Fos, P. J., Johnson, W. D., Kamali, V., Cox, R. G., Zuniga, M. A., et al. (2008). Body mass index and health related quality of life in elementary school children: A pilot study. Health Qual Life Outcomes, 6, 77.

47. Kruger, J., Bowles, H. R., Jones, D. A., Ainsworth, B. E., \& Kohl, H. W. (2007). Health-related quality of life, BMI and physical activity among US adults ( $\geq 18$ years): National Physical Activity and Weight Loss Survey, 2002. International Journal of Obesity (London), 31(2), 321-327.

48. Sawyer, M., Antoniou, G., Toogood, I., \& Rice, M. (1999). A comparison of parent and adolescent reports describing the health-related quality of life of adolescents treated for cancer. International Journal of Cancer Supplement, 12, 39-45.

49. Jeffery, A. N., Voss, L. D., Metcalf, B. S., Alba, S., \& Wilkin, T. J. (2005). Parents' awareness of overweight in themselves and their children: Cross sectional study within a cohort (EarlyBird 21). BMJ, 330(7481), 23-24.

50. Fallon, E. M., Tanofsky-Kraff, M., Norman, A. C., McDuffie, J. R., Taylor, E. D., Cohen, M. L., et al. (2005). Health-related quality of life in overweight and nonoverweight black and white adolescents. Journal of Pediatrics, 147(4), 443-450.

51. Schwimmer, J. B., Burwinkle, T. M., \& Varni, J. W. (2003). Health-related quality of life of severely obese children and adolescents. JAMA, 289(14), 1813-1819.

52. Nordyke, K., Norstrom, F., Lindholm, L., Carlsson, A., Danielsson, L., Emmelin, M., et al. (2011). Health-related quality-oflife in children with coeliac disease, measured prior to receiving their diagnosis through screening. Journal of Medical Screening, 18(4), 187-192.

53. Varni, J. W., Limbers, C. A., \& Burwinkle, T. M. (2007). Parent proxy-report of their children's health-related quality of life: An analysis of 13,878 parents' reliability and validity across age subgroups using the PedsQL 4.0 Generic Core Scales. Health and Quality of Life Outcomes, 5, 2.

54. Theunissen, N. C., Vogels, T. G., Koopman, H. M., Verrips, G. H., Zwinderman, K. A., Verloove-Vanhorick, S. P., et al. (1998). The proxy problem: Child report versus parent report in healthrelated quality of life research. Quality of Life Research, 7(5), 387-397. 